\title{
Los desplazamientos sociales en los programas de renovación urbana del Centro Histórico de Lima (1996-2016)
}

Sharo Lopez. Centro de investigación de estudios Urbanos y Territoriales URBES-LAB, Lima, Perú.

Rodrigo Paraizo. Universidade Federal do Rio de Janeiro, Rio de Janeiro, Brasil.

RESUMEN | Las condiciones de precariedad y pobreza urbana de los residentes del Centro Histórico de Lima se han profundizado debido a las reformas estatales de libre mercado implementadas en la década de 1990, entre las cuales figuran los programas de renovación urbana con inversión privada. Bajo estos programas, el Estado adjudicó al mercado la prerrogativa de resolver el problema del acceso a la vivienda. En el artículo se presentan tres casos de estudio que comprueban que estos programas, ejecutados por los gobiernos nacional y local, no han logrado revertir el problema de la vivienda tugurizada y que, además, han causado diversos tipos de desplazamiento social: directos e indirectos, en cadena, y antes, durante y después de su ejecución. Asimismo, se concluye que los tres programas estudiados fueron puntuales, pues solo se centraron en inmuebles específicos; por ende, fueron excluyentes, considerando que los beneficiarios debían cumplir un conjunto de requerimientos de la banca.

PALABRAS CLAVE | patrimonio, política habitacional, renovación urbana.

ABSTRACT | The precarious conditions of slums and urban poverty in Lima's Historic Center has worsened, mainly due to the state free market reforms implemented in the decade of 1990, including the urban renewal program that relies on private investment. Under these programs, the State granted the market the prerogative of solving the access to housing. This article introduces three case-studies that prove that urban renewal programs, implemented by the national and local governments, have not managed to reverse slumming and have generated various types of social displacement: direct and indirect, chained, before, during and after its execution. We conclude that the three analyzed programs were very specific, since they only focused on properties due to their exclusivity, therefore, being excluding, considering that the beneficiaries had to meet a set of banking requirements.

KEYwORDs | heritage, housing policy, urban renewal. 


\section{Introducción}

El área del Centro Histórico de Lima ( $\mathrm{CHL}$ ), declarado Patrimonio Cultural de la Humanidad desde 1991, contiene 649 inmuebles declarados como monumentos, 1.278 inmuebles con valor monumental, 5.199 inmuebles con valor de entorno, y 67 inmuebles urbanos monumentales (Programa Municipal para la Recuperación del Centro Histórico de Lima [prolima], 2019). Este tipo de patrimonio material inmueble está indefectiblemente ligado al patrimonio inmaterial conservado por la población, dado que corresponde a las costumbres criollas, las mismas que se concentran, principalmente, en los barrios residenciales, como Barrios Altos o Monserrate, que son los núcleos de origen de dichas tradiciones. A pesar de la intención de mantener el CHL como un centro vivo, el número de residentes ha disminuido desde el año 2000 y las brechas de desigualdad han aumentado.

De acuerdo con los datos de la Municipalidad Metropolitana de Lima (MML, 2014), el número de viviendas tugurizadas se ha incrementado durante el periodo 1996-2016, convirtiéndose en un problema. De un total de 1.140 inmuebles inspeccionados, aproximadamente se declararon 742 como inhabitables, tugurizados y/o ruinoso total, y 303 como inhabitables, tugurizados y ruinoso parcial, siendo el $90 \%$ de inmuebles calificados en situación de tugurización. En términos generales, una vivienda tugurizada es una residencia dividida, compartida, densificada y habitada por población vulnerable. Se caracteriza por encontrarse en malas condiciones físicas (además de carecer de saneamiento legal) y sin adecuado abastecimiento de servicios. El problema de la vivienda tugurizada no es nuevo; por el contrario, nace en la época republicana, cuando los dueños comenzaron a alquilar sus inmuebles sin prestar atención a su conservación (Ludeña, 2002).

Con el objetivo de hacer frente a esta problemática, en el período 1996-2016 la MML y el Ministerio de Vivienda, Construcción y Saneamiento (MvCs) desarrollaron tres programas de renovación urbana enfocados en la destugurización y erradicación de tugurios. Sin embargo, estos programas fueron insuficientes para solucionar los problemas estructurales y se caracterizaron por ser exclusivos, pues solo podían acceder al beneficio de la vivienda propia quienes contaran con el bono inicial para su calificación en el sistema financiero. En este espacio urbano, en el que la población sobrevive gracias al ambulantaje u otras labores informales, la obtención de una vivienda adecuada a través del sistema inmobiliario privado se convierte en una utopía. Contrario a lo esperado, los programas de renovación urbana han generado más impactos sociales negativos, como los desplazamientos sociales, el incremento de viviendas tugurizadas y la ampliación de la precariedad.

Las investigaciones relacionadas a los proyectos y programas de renovación urbana en la ciudad de Lima pertenecen, principalmente, al campo disciplinar de la arquitectura, con algunos aportes desde la economía y las ciencias políticas. Entre los temas más analizados se encuentran la renovación urbana en los espacios públicos (Herrera, 2005; Olivera, 2014; Rodríguez, 2014, 2018). También hay investigaciones acerca de la participación de la población en los programas de renovación urbana (Lagos, 2012; Mollo, 2018) y propuestas para lineamientos técnicos en los proyectos de renovación urbana (Álvarez \& Mendoza, 2016). En cuanto al 
análisis de los programas, Uceda (2014) documenta las experiencias del programa de renovación urbana El Porvenir (1972-1975), concluyendo que, a pesar de haber contado con decisión política para su puesta en marcha, solo se llegó a construir algunos edificios.

A estas investigaciones se suman las que analizan el inquilinato de la vivienda tugurizada (Decker, 2015) y la factibilidad de construcción de la vivienda de interés social en el CHL (Lozada, 2014). Además, se ha investigado y analizado la supuesta gentrificación en el cHL y sus implicancias (Castillo, 2015; Roldán, 2017). También, existen investigaciones que se han ocupado de analizar la precariedad urbana y los desalojos en el chl (Dammert Guardia, 2018). Como es visible, hay un vacío teórico y analítico acerca del impacto de los proyectos de renovación urbana respecto de los ocupantes de los inmuebles intervenidos en el cHL. En esta línea, el artículo cobra relevancia al analizar un fenómeno que no ha sido estudiado por la academia ni identificado por las autoridades. Además, revisa los proyectos de renovación urbana con una mirada crítica, resaltando la importancia de la permanencia de la población residente y la toma de conciencia sobre fenómenos asociados. Mediante el estudio de casos, se examinan los desplazamientos sociales causados por los proyectos de renovación urbana en el cHL durante el periodo 1996-2016.

El documento se encuentra estructurado en cinco partes. Primero, se realiza una exploración teórica sobre los desplazamientos sociales en las áreas centrales; en segundo lugar, se explica la metodología aplicada en la investigación. En la tercera parte, se analiza la renovación urbana como mecanismo desplazador en un contexto de reformas neoliberales, propias de la producción capitalista del espacio urbano central. En un cuarto momento, se presentan los casos de estudio y los principales hallazgos y, finalmente, se expone las conclusiones y reflexiones.

\section{Aproximaciones teóricas}

Los desplazamientos sociales en América Latina (AL), generalmente, son analizados relacionándolos con los procesos de gentrificación (Crestani, 2015; Martí-Costa et al., 2016). Sin embargo, al examinar este fenómeno como un efecto secundario, puede ocurrir que se invisibilice factores y los mecanismos propios de la producción urbana de AL. En este contexto, se entiende por desplazamiento social el proceso de expulsión que implica movilizar o cambiar a alguien de su lugar de residencia, voluntaria o involuntariamente. Asimismo, los desplazamientos, por su origen, son entendidos como condición o efecto. Como condición: cuando es necesario trasladar a un colectivo para el desarrollo de proyectos urbanos a escala y para el desarrollo económico-competitivo. Como efecto: por políticas públicas, desregulación inmobiliaria, construcción de proyectos urbanos y vivienda, que generan ambientes no aptos para residir (Delgadillo, 2015).

Se entiende por desplazamientos sociales en las áreas centrales (voluntarios o involuntarios), aquellos asociados a políticas urbanas desarrolladas como "una política estratégica para expulsar ciertas prácticas sociales (...) no deseadas, impidiendo que determinada población pueda consumir y apropiarse de partes específicas de la ciudad" (Janoschka \& Sequera, 2014, p. 4), y restringiéndose con ello el derecho a 
la ciudad y a la vivienda. Según Janoschka (2016), en AL los desplazamientos tienen cuatro dimensiones inherentes: desplazamiento como proceso material, como proceso político, como proceso simbólico y como proceso psicológico. Para el caso del CHL, en donde no se encuentra regulado el derecho a la vivienda, los desplazamientos mutaron de política estratégica a práctica consumada de hacer ciudad.

El desplazamiento, al ser ejecutado por el Estado con ventaja hegemónica, restringe la permanencia y las prácticas alternativas que promueven un lugar adecuado para vivir. En este sentido, se sostiene en políticas y normas que impiden ciertas actividades sociales, y está asociado a cinco funciones fundamentales de la reproducción social: "la vivienda, el suelo, los bienes comunes (entre los cuales destaca el espacio público), la movilidad, y la propia constitución y autonomía del sujeto" (Janoschka, 2016, p. 38).

Existen distintos tipos de desplazamientos sociales, caracterizables como involuntarios forzosos (DIF)-desalojos, involuntarios coaccionados (DIC), y voluntarios por presión (DVP) (Delgadillo, 2015), los cuales tienden a ser ejemplares o ligeros (Carman, 2015). En el estudio de los desplazamientos sociales es relevante reconocer, además, las formas de desplazamiento. En este sentido, Marcuse $(1985,1986)$ propone estudiar el desplazamiento directo del último residente, el desplazamiento directo en cadena, el desplazamiento excluyente y la presión de desplazamiento. Por otro lado, también es importante reconocer quién es desplazado; es decir, su situación económica, empleo, tipo de empleo, estructura familiar, etcétera, así como conocer la configuración espacial del lugar adonde fue desplazado.

El reconocimiento de la ocurrencia de desplazamientos en los centros urbanos está supeditado a dos situaciones: la condición de abandono de los centros urbanos y la necesidad de datos cuantitativos que demuestren el desplazamiento de las personas. Ambas se soportan para invalidar el fenómeno. Ante ello, con base en la experiencia de México, Delgadillo (2015) establece algunas recomendaciones metodológicas: análisis de las estadísticas poblacionales, análisis de políticas y normas sobre el espacio público, entrevistas, entre otras.

También es importante entender los mecanismos y factores que generan los desplazamientos. En ese sentido, González et al. (2015) y Delgadillo (2015) establecen cinco categorías de análisis: i) Mecanismo dinamizador del desplazamiento, el cual busca distinguir los mecanismos involucrados (económico, acoso inmobiliario y político coercitivo); en esta investigación solo se analiza el mecanismo político coercitivo; ii) Proceso de desplazamiento, que se enfoca en los diferentes ritmos y estadios del fenómeno, los cuales pueden ser instantáneos o progresivos; iii) Configuración espacial del desplazamiento, respecto del cual se analiza la dinámica espacial del fenómeno, lo que permite detectar los cambios del paisaje urbano; iv) Grupo social sujeto a riesgo de desplazamiento, que permite conocer al sujeto social desplazado, su asociación con el uso de suelo (habitacional o comercial), así como su organización ante estos procesos (juntas vecinales); v) Finalmente, perceptibilidad del desplazamiento, que mide la visibilidad de los procesos y permite evidenciar el grado de obligatoriedad.

Además de las concepciones teóricas de los desplazamientos sociales, es preciso entender que este fenómeno no es nuevo; por el contrario, forma parte de la 
producción del espacio urbano capitalista de las áreas centrales (Lefebvre, 1968). En ese sentido, es importante contextualizar el fenómeno para el caso peruano. Desde la década de 1980, AL incorporó un conjunto de reformas neoliberales caracterizadas por la disminución de funciones del Estado y el posicionamiento del libre mercado. En el Perú, estas reformas fueron implementadas en el gobierno autocrático de Alberto Fujimori (1990-2000), y consistían principalmente en la privatización de empresas públicas; la relajación de los servicios de transporte urbano mediante la concesión de nuevas rutas y la importación de automotores usados de diverso tamaño y condición (Contreras \& Cueto, 2018); ajuste en la deuda externa, es decir, la solicitud de nuevos créditos, la atracción de inversión privada e incremento de exportaciones; y el ajuste del gasto fiscal, expresado en la reducción de inversiones en programas sociales, lo que impactó principalmente en la educación, salud pública y el apoyo a la investigación científica universitaria.

En el ámbito urbano, la producción del espacio urbano pasó a manos del sector empresarial, que produce la ciudad en términos de rentabilidad urbana, concentrándose en sectores que permitirán mayores réditos económicos, profundizando la desigualdad, la fragmentación y la pobreza urbana. La producción del espacio urbano central en la ciudad del Lima, desde la década de 1990, estuvo abocada a la recuperación de espacios públicos, peatonalización de calles, pintura de fachadas, restauración de balcones coloniales y republicanos, expulsión de comerciantes ambulantes y la consolidación de una nueva imagen urbana del CHL. Ante ello, la población encontró maneras de acceder a la vivienda mediante las invasiones, posesiones, alquiler, entre otros (Dammert Guardia, 2018).

\section{Aproximaciones metodológicas}

El enfoque de la investigación cualitativa permite entender la problemática con mayor profundidad que aproximaciones cuantitativas, y nos ayuda a conocer detalles más precisos sobre los procesos. De las entrevistas se pudo conocer que los desplazamientos no solo se dieron al inicio de los programas de renovación urbana, sino en diversas etapas del proceso. Asimismo, permitieron identificar las particularidades del fenómeno en el CHL y las percepciones de los diversos actores.

Para el análisis de la relación entre programas de renovación urbana y los desplazamientos sociales de la población del CHL, se realizaron 13 entrevistas semiestructuradas con los siguientes grupos: vecinos, población desplazada, ${ }^{1}$ dirigentes vecinales y autoridades municipales. El trabajo de campo se realizó en dos etapas, mayo del 2016 y diciembre del 2016. Para el trabajo de campo se contactó a profesionales ligados a temas del $\mathrm{CHL}$, quienes nos presentaron a los dirigentes vecinales. A partir de ello se realizó una estrategia de "bola de nieve", la cual permitió contactar a los demás entrevistados. En cuanto a los casos, se seleccionó tres, cada uno correspondiente a periodos municipales: los gobiernos de Andrade (1996-2003), Castañeda (2004-2011) y Villarán (2011-2014) (Figura 1).

1 De acuerdo con los términos del consentimiento libre e informado (TCLI), los nombres de los entrevistados quedarán en reserva; solo se mostrarán etiquetas. 
Por otro lado, se analizaron datos y documentos proporcionados por PROLIMA, de la Subgerencia de Renovación Urbana de la mmL (2014); archivos de la Biblioteca Municipal y archivos de la Biblioteca del Consejo de Ministros. También se usaron documentos producidos por medios de comunicación (periódicos, TV e internet). Además, se realizó un registro fotográfico de los inmuebles y se lo comparó con las fichas inmobiliarias de 1996.

A fin de explicitar, analizar e identificar los desplazamientos sociales, se adaptaron las categorías desarrolladas por González et al. (2015) y Delgadillo (2015) a las especificidades del CHL, obteniéndose las siguientes: condición de los inmuebles, grupo social sujeto a riesgo, proceso del desplazamiento (se estudia la temporalidad y territorialidad) y perceptibilidad. Estas categorías son construidas con la información recolectada (cualitativa y cuantitativa). En ese sentido, los resultados se encuentran organizados de acuerdo con estas categorías.

\section{FIgURA I | Temporalidad de los desplazamientos en el CHI}

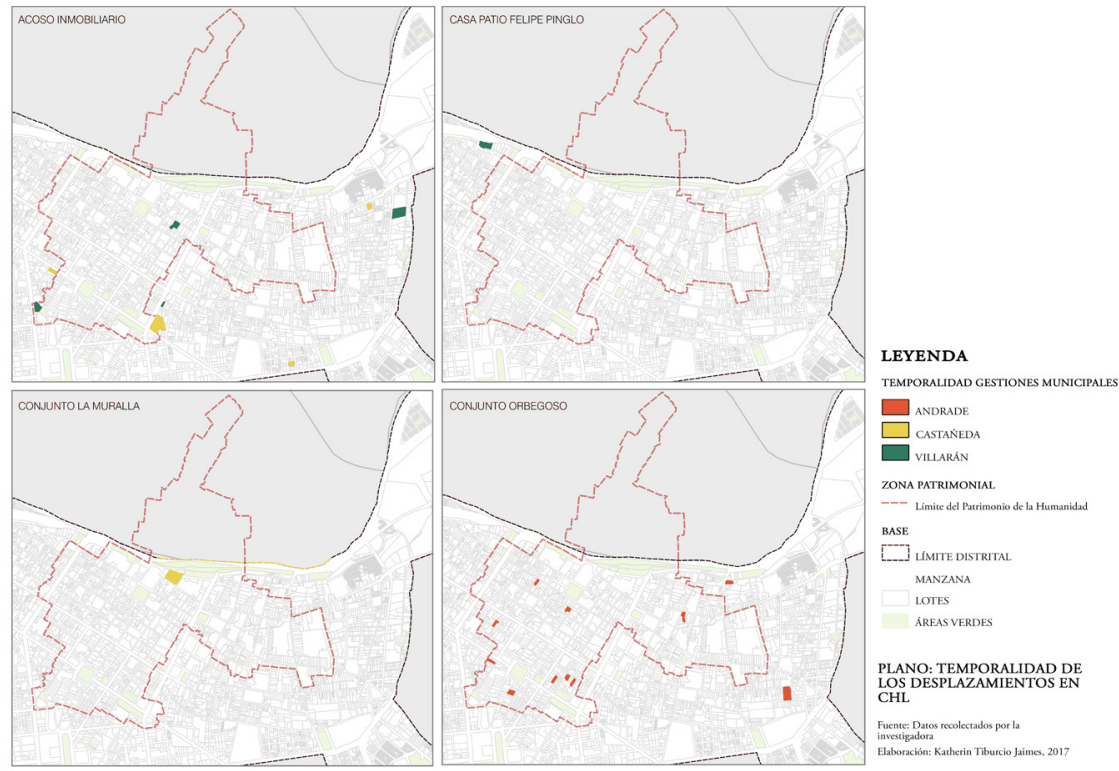

FUENTE: ELABORACIÓN PROPIA

Las reformas urbano-neoliberales:

la renovación urbana en el Centro Histórico de Lima (1996-2016)

La vivienda para la producción capitalista del espacio urbano pasó a ser desarrollada por el sector inmobiliario y el sistema financiero (Harvey, 2005; Rolnik, 2015), en tanto el Estado asumió el papel de gestor. En el Perú, el ajuste estructural influyó en la eliminación del derecho a la vivienda establecido en la Constitución Política de 1979 y el establecimiento del paradigma de la vivienda propia (De Soto, 
1986). En ese marco, se emitió el Decreto Legislativo 696 (Ley de Promoción a la Inversión Privada en Acciones de Renovación Urbana de 1991), y su reglamento fue aprobado por el Decreto Supremo 11-95-мтс. Ambos documentos declaran de "necesidad, utilidad pública e interés social la inversión privada en acciones de renovación urbana a nivel nacional" (Ministerio de Transportes y Comunicaciones [MTC], 1991). Así, la responsabilidad y la regulación de las políticas habitacionales fueron cedidas al mercado (privado y financiero).

Según la normativa, el sector privado se encargaría de la recuperación de los centros históricos y tugurios para sustituirlos por proyectos de alta densidad y altura. En tanto, las municipalidades tendrían como competencias la promoción, orientación, aprobación y control de las acciones de renovación, y la identificación de áreas de tratamiento con fines de renovación urbana, para lo cual era necesario tener en cuenta los siguientes requerimientos: "el grado de deterioro urbano y la población involucrada, los casos de alto riesgo por seguridad física, la calidad de edificios y espacios de valor histórico monumental, las densidades poblacionales mínimas a lograrse en los proyectos" (MTC, 1991, art. 5).

Contrario a promover el derecho a la vivienda, que protege al residente ante desalojos, el Decreto Supremo 11-95-MTC reconoce el derecho a residencia de los ocupantes de los predios por renovar, entendido como el derecho de realojamiento de los ocupantes. Con tal fin, "los ejecutores concertarán con los pobladores las condiciones de su permanencia en las áreas renovadas o de su reubicación en otras áreas, si no pudieran asumir la diferencia de costo derivada de las acciones de renovación" (MTC, 1991, art. 7). En resumen, este decreto condiciona la permanencia de la población residente de las áreas sujetas a intervención, a su posibilidad de asumir los costos que implican la construcción de nuevos conjuntos habitacionales. Asimismo, concede al sector privado y/o público el criterio de concertar o no con la población el destino de su realojamiento, así como el de temporalidad y el de las condiciones de construcción y financiamiento. Es decir, no se incluye la participación de la población.

El derecho a residencia o el desplazamiento concertado genera una contradicción entre la conceptualización del CHL y su forma de ser intervenido. De acuerdo con el Plan Maestro del Centro de Lima de 1998: "El Centro Histórico de Lima es una estructura socioeconómica, espacial y cultural (...) que constituye expresión de la creatividad cultural de la comunidad local y mantiene las características y calidades de vidas propias de núcleos urbanos en actividad" (MML, 1998, art. 25). Entonces, la calidad de centro histórico es conferida tanto por las estructuras testimoniales como por la población que mantiene vivo este núcleo. El desplazamiento de la población residente a otras áreas urbanas y el desalojo repercutirían directamente en el tipo de chl que se pretende conservar y construir, es decir, un centro vivo.

Entre los años 2009 y 2010, durante la segunda presidencia de Alan García Pérez y la alcaldía de Luis Castañeda Lossio -gobiernos que priorizaron la inversión privada- se aprobó la Ley de Saneamiento Físico Legal de Predios Tugurizados con Fines de Renovación Urbana 29415-2009, y su reglamento, Decreto Supremo 011-2010-viViendA, en el cual se declara de necesidad pública y de preferente interés social y nacional la renovación urbana y destugurización con fines de vivienda. 
Según este documento, la renovación urbana tendría por finalidad la eliminación de tugurios, hacinamiento y demás situaciones de inhabitabilidad (Ministerio de Vivienda, Construcción y Saneamiento [MVCs], 2009) mediante acciones que resulten necesarias para mantener, mejorar y modernizar las viviendas existentes. Según la norma, la renovación urbana constaría de dos etapas: la primera, de saneamiento legal; y la segunda, de saneamiento físico.

La primera etapa de la renovación urbana, según la Ley 29415, pretende subsanar el vacío legal en el que se encuentran los inmuebles tugurizados del CHL. Esta norma promueve dos instrumentos que procuran mayor eficiencia para el reconocimiento de propiedad: la declaración administrativa de abandono y la prescripción adquisitiva de dominio. Los inmuebles en propiedad del Estado solo pueden aplicar a la segunda herramienta. Una vez declarado en abandono, el inmueble pasa a ser propiedad de la municipalidad, y esta, a su vez, transfiere la propiedad a la asociación de vecinos. Asimismo, con la promulgación de esta ley, las familias se beneficiarían al "sanear legal y físicamente los inmuebles que ocupan, ser sujetos a créditos y obtener facilidades de pago para la renovación y modernización de sus viviendas, elevando así sus niveles de vida" (MVCs, 2009, p. 14). De acuerdo con esta norma, las familias con posibilidades y que cumplan los requisitos del sistema crediticio podrán adquirir sus viviendas.

La segunda etapa, referida al saneamiento físico, procura revertir el estado de tugurización, inhabitabilidad y las demás condiciones de los inmuebles en riesgo, pero tal proceso solamente será posible cuando el inmueble cuente con título de propiedad (MvCs, 2009). Para la eliminación de la tugurización, la ley promueve programas de renovación urbana que constituyen "instrumentos de gestión urbanística, que comprenden el desarrollo y ejecución de obras a través de proyectos inmobiliarios y de infraestructura" (MVCs, 2009, art. 26). Estos programas son aprobados e impulsados por las municipalidades conforme a sus planes de desarrollo urbano (PDU).

La sutil diferencia entre las normativas señaladas es que la Ley 29415 tiene como foco principal el saneamiento legal de los inmuebles tugurizados a favor de las asociaciones de vivienda, siempre y cuando el propietario entre en consenso de ejecutar viviendas sociales. Y la Ley 696, por su parte, tiene como principal foco la promoción de la inversión privada. A pesar de ello, ambas leyes formulan y responden a una política habitacional por la que cada residente debe ser propietario (Figura 2).

Primer caso: El programa de destugurización del Centro Histórico de Lima (1996) En este periodo, el alcalde Alberto Andrade se concentró en la implementación del programa de recuperación urbana del CHL y la reubicación de los comerciantes ambulantes, sin intervenir ni proponer programas para las viviendas tugurizadas. Este programa de destugurización fue desarrollado por el gobierno central, desde la presidencia y el MTC, siendo el primer programa realizado en Barrios Altos, por lo cual es relevante comprender su impacto urbano y social.

El Decreto Supremo 038-96-PCM (Presidencia del Consejo de Ministros), del 20 de agosto de 1996, sustentó y reglamentó el programa de destugurización. Con esta norma se autorizó a la Empresa Nacional de Edificaciones (ENACE) y al Fondo 
Nacional de Vivienda (FONAVI) a destugurizar doce inmuebles de propiedad de la Sociedad Beneficencia Pública de Lima (SBLM). El decreto señala que estos inmuebles se encuentran en condición de tugurios: "dichos inmuebles han sido declarados en riesgo de colapso (...) requieren ser rehabilitados, reconstruidos o demolidos" (MTC, 1996).

La política de vivienda aplicada por el programa de destugurización se enfocó en acciones puntuales, de resultados inmediatos y sin estudio previo. A pesar de la intención de mejorar las condiciones de habitabilidad en el CHL, los resultados muestran todo lo contrario. De los doce inmuebles intervenidos, diez siguen siendo quintas-corralones en donde las condiciones de inhabitabilidad persisten. Asimismo, se puede verificar que la población en riesgo de desalojo se ha incrementado. Esto último no solo está relacionado con la propiedad de los inmuebles sino, también, con la actuación del gobierno local, nacional y de la SBLM.

\section{Segundo caso: El conjunto habitacional La Muralla (2003-2010)}

El conjunto La Muralla forma parte del Primer Programa de Renovación Urbana (PPRU) desarrollado en el CHL. El gobierno municipal de Luis Castañeda Lossio (2003-2010) fue el responsable de su formulación, diseño y ejecución. La Memoria de Gestión (2003-2010) se refiere al conjunto de la siguiente manera:

No es sólo una nueva vivienda, es una nueva forma de vida. Uno de los objetivos y
ejes fundamentales (...) fue trabajar por la revalorización social y económica de las
familias de extrema pobreza y a la recuperación inmobiliaria, constituyéndose en
el organismo promotor del desarrollo. (Empresa Municipal Inmobiliaria de Lima
[EmILIMa], 2010, p. 30 )

El PPRU consistía en la recuperación urbana de la manzana llamada El Rastro y La Soledad, donde se localizaban siete inmuebles de distinto valor patrimonial. En ese contexto se restauraron inmuebles históricos y con valor monumental, y se construyó el conjunto La Muralla para los residentes de la manzana intervenida. A diferencia del primero, este programa habitacional incluyó la participación vecinal en los trabajos de construcción, la cual consistía en el pago de la vivienda con horas de trabajo.

Tercer caso: El conjunto habitacional Los Patios de Felipe Pinglo (2010-s.f.)

El programa de construcción del conjunto habitacional Los Patios de Felipe Pinglo, perteneciente a la segunda etapa del PPRU, también fue formulado durante el gobierno del alcalde Castañeda (2003-2010) e implementado por los gobiernos municipales posteriores. El conjunto se encuentra localizado en el barrio de Monserrate, en el CHL, y el programa se realizó en tres inmuebles de propiedad de la MML.

El conjunto habitacional fue proyectado con el nombre de Cañete 100, con las mismas características del realizado durante la primera etapa del PPRU. Sin embargo, durante el gobierno de Susana Villarán (2011-2014) se cambió la naturaleza del proyecto y pasó a ser construido como alojamiento temporal. En la actualidad, el conjunto se encuentra en estado de abandono por motivos judiciales y económicos. En consecuencia, no se tiene el análisis pos retorno de la población beneficiada, es decir, las familias del PPRU y las que habitaban el terreno. 
FIGURA 2 | Políticas públicas relacionadas

MECANISMO DINAMIZADOR: Políticas y proyectos públicos

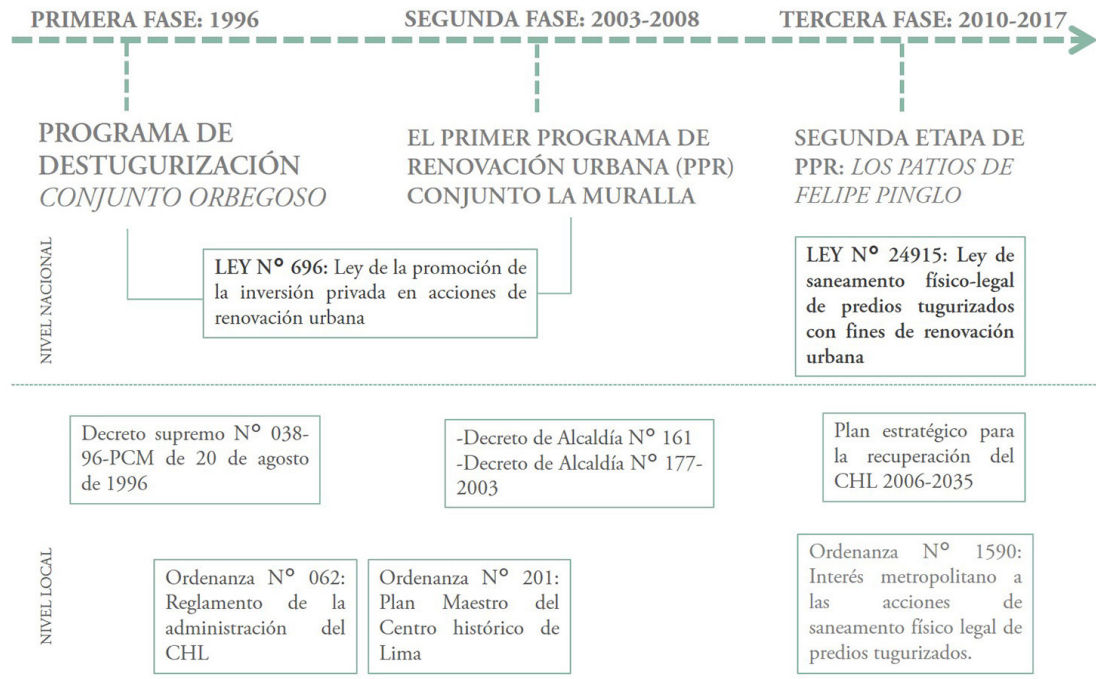

FUENTE: ELABORACIÓN PROPIA

\section{Discusión}

\section{Los desplazamientos sociales en el Centro Histórico de Lima (1996-2016)}

Los desalojos históricos y los procesos de desplazamiento en el marco de la producción capitalista del espacio urbano difieren en los mecanismos aplicados. En la actualidad se hacen presentes mecanismos como la renovación urbana, revitalización y otras formas de intervención en áreas degradadas, los cuales tienen como característica principal intervenir un área urbana de manera puntual (Lopez, 2017). Además, estas formas de intervenir la ciudad generan la diferenciación entre la población residente beneficiada y la no beneficiada; es decir, son políticas urbanas focalizadas. Así, las políticas urbanas neoliberales, en vez de resolver problemas presentes durante largos periodos históricos, los agudizan y generan desplazamientos, desigualdad urbana e injusticia social (Contreras, 2017).

De acuerdo con lo desarrollado en el ítem anterior, las dos leyes principales que rigen la renovación urbana en el Perú son la Ley 696 y la Ley 29415. Ambas fueron adaptadas en la normativa de la MML, acríticamente y sin ninguna mejora. En este sentido, las acciones realizadas con los programas de renovación urbana no contemplan acciones con sentido social ni consideran el derecho a la vivienda, pues se rigen por el paradigma de la vivienda propia y la rentabilidad urbana.

La triangulación de la información de los casos será realizada en concordancia con las categorías de análisis: condición del inmueble, grupo social sujeto a riesgo, proceso del desplazamiento y perceptibilidad. 


\section{Condición de los inmuebles}

Para el desarrollo de programas de renovación urbana, una de las primeras condiciones es que los inmuebles tengan una declaración de tugurio, ya que ello justifica la mejora de condiciones de las viviendas tugurizadas. Sin embargo, no todos los inmuebles intervenidos a partir de las dos leyes comentadas, y considerados en este estudio, se encontraban en malas condiciones de habitabilidad (EMILIMA, 2014a). En el primer caso de estudio, once inmuebles se encontraban en mal estado de conservación. En el segundo caso, de los siete inmuebles, solamente uno se encontraba en mal estado. En el tercer caso, todos se encontraban en mal estado. También es oportuno acotar que la mayoría de los inmuebles contaba con categoría de valor monumental (vM) y monumento histórico $(\mathrm{MH})$ (Tabla 1), entendido que los inmuebles con categoría VM y MH tienen una especial atención de parte del gobierno local y del Ministerio de Cultura, que lleva a dejar de intervenir en inmuebles del entorno.

En cuanto a la localización de los inmuebles intervenidos, en el primer caso, nueve inmuebles se encuentran en el Damero de Pizarro (zona de mayor afluencia turística) y tres se encontraban en Barrios Altos (BA), efectuándose la construcción de nuevos predios en dos de los tres inmuebles de BA. En el segundo, la manzana intervenida se encuentra en el Damero de Pizarro, a una cuadra del Palacio de Gobierno; y la tercera, en el barrio de Monserrate. Como resultado, los programas no intervinieron en inmuebles que realmente necesitaran una mejora de condiciones, sino en aquellos entre cuyas características estaban el ser de propiedad estatal, tener localización exclusiva (al encontrarse en zonas de mayor atractivo turístico) y contar con al menos valor monumental.

\section{Grupo social sujeto a riesgo}

La situación económica de las familias habitantes de los inmuebles intervenidos era diversa. De acuerdo con el análisis de documentos y entrevistas, es posible verificar que, para los tres casos, los ingresos oscilaban entre 600 y 800 nuevos soles. Según las entrevistadas 2 y 3 (com. pers., 9 diciembre 2016), el proveedor por familia era diverso. Para el caso del programa de destugurización, en su inmueble predominaban las jefas de familia, la actividad principal era el comercio ambulatorio u otro tipo de actividad informal y, en todos los casos, las familias alquilaban u ocupaban los predios, ya que los propietarios son la MML y la sBLM. El alquiler oscilaba entre 15 y 45 nuevos soles (Tabla 2).

Durante el proceso de recolección de datos fue posible verificar que no existe un número preciso de familias desplazadas, cantidad que varía de acuerdo con la fuente de información. Para la investigación se utilizó como base los datos de la MML. En el primer caso de estudio, 212 familias fueron desalojadas hacia el conjunto Orbegoso. En el segundo caso, eran 112 las familias habitantes de los inmuebles, y las familias beneficiarias fueron 90; del total de familias, $60 \%$ era población adulta, 33\% menores de edad y, el resto, personas de la tercera edad (Lagos, 2012). En el tercer caso, habitaban 26 familias en los tres inmuebles intervenidos, de las cuales 10 familias fueron a alojamientos temporales y 16 se quedaron a vivir en el centro. Los tres casos contaban con asociaciones vecinales, es decir, interlocutoras entre familias y gobierno municipal. Solo el segundo caso presenta dos asociaciones de vecinos (Tabla 2). 

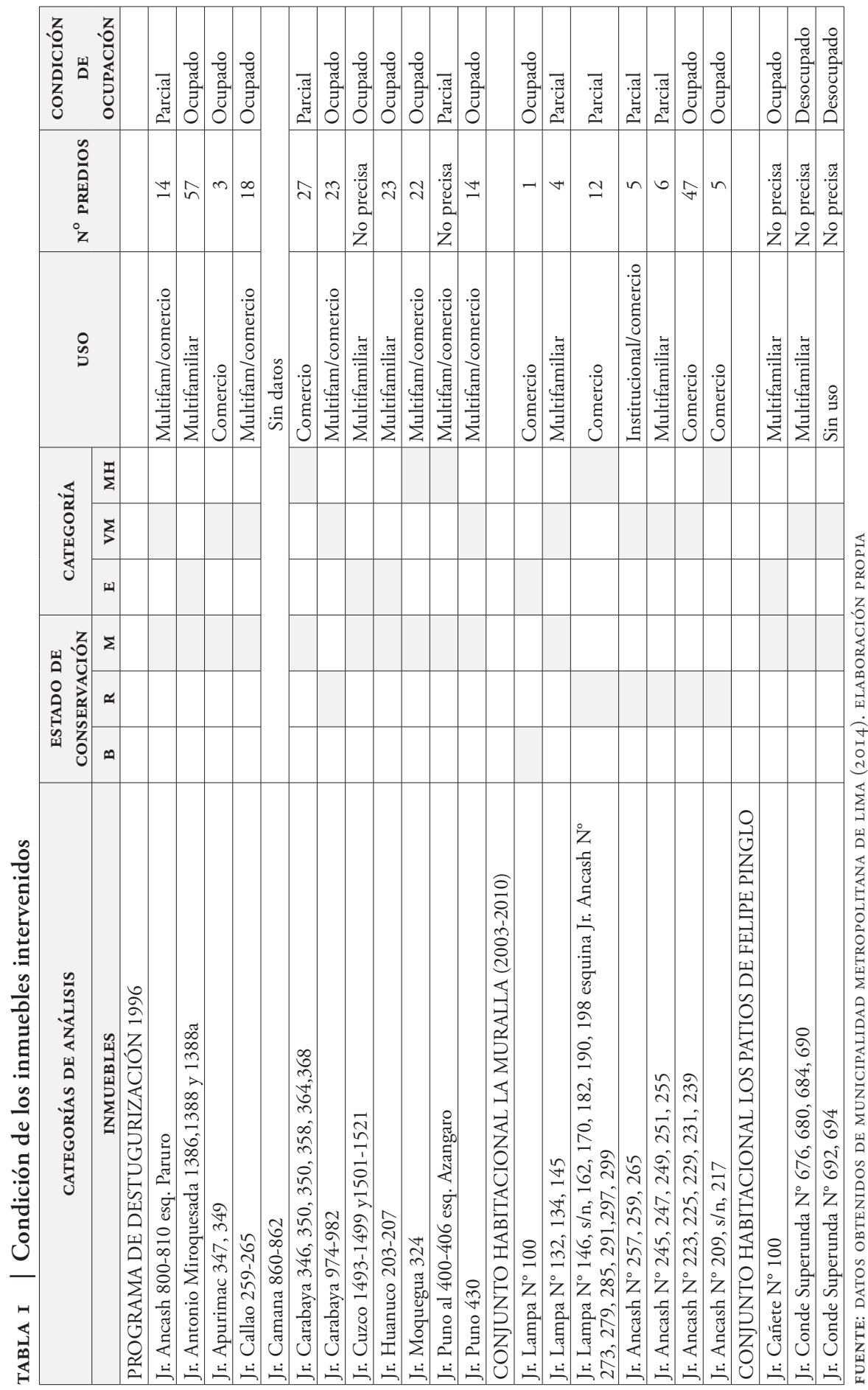


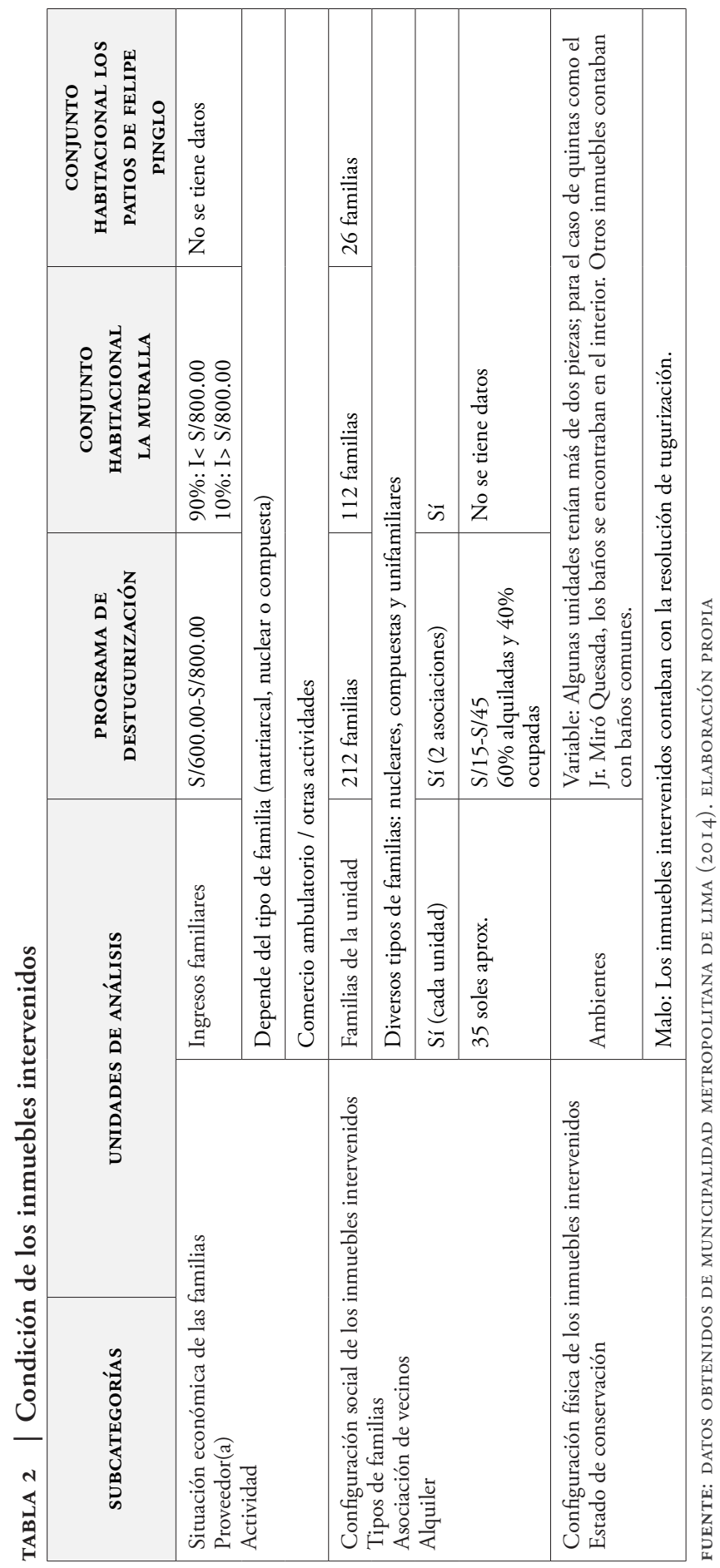




\section{El proceso de desplazamiento}

Los tres casos estudiados presentan diferentes procesos de desplazamiento. En la primera etapa, los tres proyectos siguen los protocolos formulados por las leyes de renovación urbana: encuestas e inspección física. Aparte, en los dos proyectos pertenecientes a los programas de renovación urbana, los residentes fueron llevados a alojamientos temporales en los distritos de Barranco y Surco, lejanos al CHL.

En el primer caso, el proyecto contemplaba la salida de los residentes de los doce inmuebles y su reubicación en el conjunto Gran Mariscal Orbegoso, que se encuentra en el límite oeste del Cercado de Lima, aproximadamente a 10 kilómetros de Barrios Altos. De acuerdo con la entrevistada 1, hija de un dirigente vecinal del inmueble ubicado en el Jr. Miró Quesada (hoy, Santa Rosa), los residentes no fueron informados sobre su relocalización, sino tan solo fueron encuestados sobre su situación económica:

No, no nos llegó ningún documento que nos advirtiera sobre el traslado; entonces ellos lo consideran con un desalojo, porque llegan un día tranquilo con un arsenal de camiones por todo Miró Quesada (...). De repente entraron gente que había contratado la Municipalidad (...) entonces se metían a tu casa (...). Fue algo espantoso (...). Estábamos de acuerdo con que nos trasladen, pero no de esa manera.

(Entrevistada 1, com. pers., 12 mayo 2016)

El proceso de desplazamiento de la población fue directo, calificable como desalojo. Según lo comentado por las entrevistadas, la llegada al nuevo lugar fue "chocante", pues no sabían qué les esperaba en las nuevas viviendas, como tampoco en el contexto urbano en que se situaban. Los entrevistados mencionaron que, de haber sido informados antes del desplazamiento, lo habrían aceptado, pero que probablemente sus vecinos no tendrían la misma respuesta. Las razones serían el desconocimiento del proceso, el contexto en que se dio, que los servicios no eran asequibles en el lugar de destino y que la zona era considerada peligrosa.

Los entrevistados mencionaron que después de establecidos en el conjunto habitacional Orbegoso, la SBLM les otorgó un cronograma de pagos mensuales para la adquisición de los departamentos, pagos que, sin embargo, no eran acordes a sus ingresos. De acuerdo con el Decreto Supremo 038-96-PCM, el costo de los departamentos adjudicados-vendidos estaba establecido por la SBLM, así como la tasa de interés y plazo de pago, con base en los términos de los convenios con la UTEfonAVi y ENACE. Asimismo, en el Decreto 038 se menciona: "Los ocupantes de los inmuebles citados en el artículo 2 (...) que no se acojan al sistema de adjudicación aprobado por el presente Decreto Supremo, deberán desocupar dichos inmuebles en un plazo que se fijará mediante notificación notarial” (MTC, 1996, art. 5).

En este sentido, la entrevistada 1 (com. pers., 12 mayo 2016) mencionó que algunos de sus vecinos, incluida su familia, no lograron pagar las cuotas mensuales establecidas por la SBLM. Ante ello, estas familias están en proceso judicial de desalojo con la SBLM. Asimismo, hubo entrevistados que afirmaron que algunas familias se retiraron debido a la presión del desalojo. Una de las entrevistadas proporcionó un documento suscrito por la SBLM, con fecha 1 de agosto del 2013, en el que se indica el término de contrato. La dinámica corresponde al tipo de desplazamiento 
en cadena (Marcuse, 1985), debido a que hubo un traslado posterior al proceso de desalojo instantáneo.

En el segundo caso, el proyecto tuvo una duración de siete años. En su primera etapa, la Gerencia de Participación Vecinal, de la MML, evaluó a la población residente mediante encuestas socioeconómicas, las condiciones de las viviendas tugurizadas y las de los monumentos habitados. Con este estudio social y físico se definió el grupo social beneficiario: de las 112 familias que habitaban la manzana, se seleccionó a 90 familias, y se determinó los inmuebles que serían protegidos y el área de construcción. Los inmuebles restaurados se convirtieron en museos y locales institucionales.

La segunda etapa del proyecto consistió en el traslado de las familias beneficiarias a los alojamientos temporales en la zona sur de Lima (Barranco y Surco). La mayoría de las familias permaneció en estos ambientes durante la construcción de las nuevas viviendas. De acuerdo con las entrevistadas 2, 3 y 4 (com. pers., 9 y 16 diciembre 2016), los primeros años en el alojamiento temporal se caracterizaron por una comunicación constante con la municipalidad, se generaron talleres ocupacionales y otras actividades, pero luego estas atenciones fueron disminuyendo. La tercera etapa, el retorno de las familias al CHL, estuvo caracterizada por emoción e incertidumbre: según la entrevistada 2 (com. pers., 9 diciembre 2016), ex dirigente vecinal del conjunto habitacional, algunos de los beneficiarios tuvieron que quedarse en los alojamientos temporales, pues se redujo el número de departamentos debido a hallazgos arqueológicos en el sitio de destino.

En el caso del conjunto habitacional La Muralla convergen diferentes tipos de desplazamientos, los cuales no son inmediatos, sino graduales y en diferentes estadios. En la primera etapa, durante la evaluación de beneficiarios y no beneficiarios, se dieron dos tipos de desplazamiento: voluntario por presión (19 familias) o desalojo pacífico, y desplazamientos involuntarios forzosos (tres familias) o desalojos. Ambos tienen como mecanismo dinamizador la renovación urbana y la aplicación de políticas coercitivas. En la segunda etapa, durante la aplicación de créditos financieros a los beneficiarios, se excluyó a las familias que no contaban con la cuota inicial. Estas familias permanecieron en los alojamientos temporales durante quince ańos, consolidándose como desalojados del conjunto La Muralla y su antiguo barrio.

Las setenta familias del conjunto La Muralla, en su retorno al CHL, lo encontraron recuperado y en mejores condiciones, más seguro; viven cerca de monumentos históricos y museos importantes. Asimismo, estas familias mantuvieron sus actividades laborales y sociales. Sin embargo, de acuerdo con las entrevistadas 3 y 4 (com. pers., 9 y 16 diciembre 2016), tienen que hacer frente a mayores gastos con los mismos sueldos; por ejemplo, mencionan que la población incurre continuamente en moras y deuda por arbitrios, lo cual genera que la población vea el inmueble como productor de ingresos, llegando a alquilar o vender sus propiedades. Con ello también se producen desplazamientos voluntarios.

Respecto a la población de los alojamientos temporales en el distrito de Surco, debieron modificar sus hábitos de consumo, laborales y sociales. En definitiva, es la población que ha pasado por más eventos de violencia simbólica (Janoschka, 2016, p. 42), pues, incluso, incrementaron sus gastos en servicios básicos y alimentación, ya que Surco es un distrito de población de renta media y alta. 
Las dinámicas de desplazamiento están relacionadas con el desplazamiento excluyente y la presión de desplazamiento (Marcuse, 1985). El primero hace referencia a la pérdida de las zonas y viviendas que antes eran accesibles para la población, que es el caso de las nueve familias de Surco. El segundo hace referencia a la expulsión de los residentes (de manera indirecta) por el cambio de entorno social, que es el caso de las familias que están saliendo del conjunto La Muralla.

Respecto del tercer caso, la segunda etapa del proceso de renovación urbana en el CHL se inició durante las elecciones presidenciales del 2010. El alcalde Castañeda se encontraba en campaña presidencial, lo que aceleró la elaboración del proyecto y las primeras etapas del programa. De acuerdo con las entrevistadas, la población que residía en los terrenos municipales del proyecto ascendía a 26 familias, de las cuales solo nueve fueron al alojamiento temporal de Surco, en tanto las demás familias se encuentran residiendo, aún, en inmuebles de la SBLM. La reactivación del conjunto habitacional determinará el rumbo de las familias que se encuentran en los alojamientos temporales. En tanto no suceda, no se podrá establecer un juicio concluyente (Figura 3).

\section{FIGURA 3 | Proceso de desplazamiento}

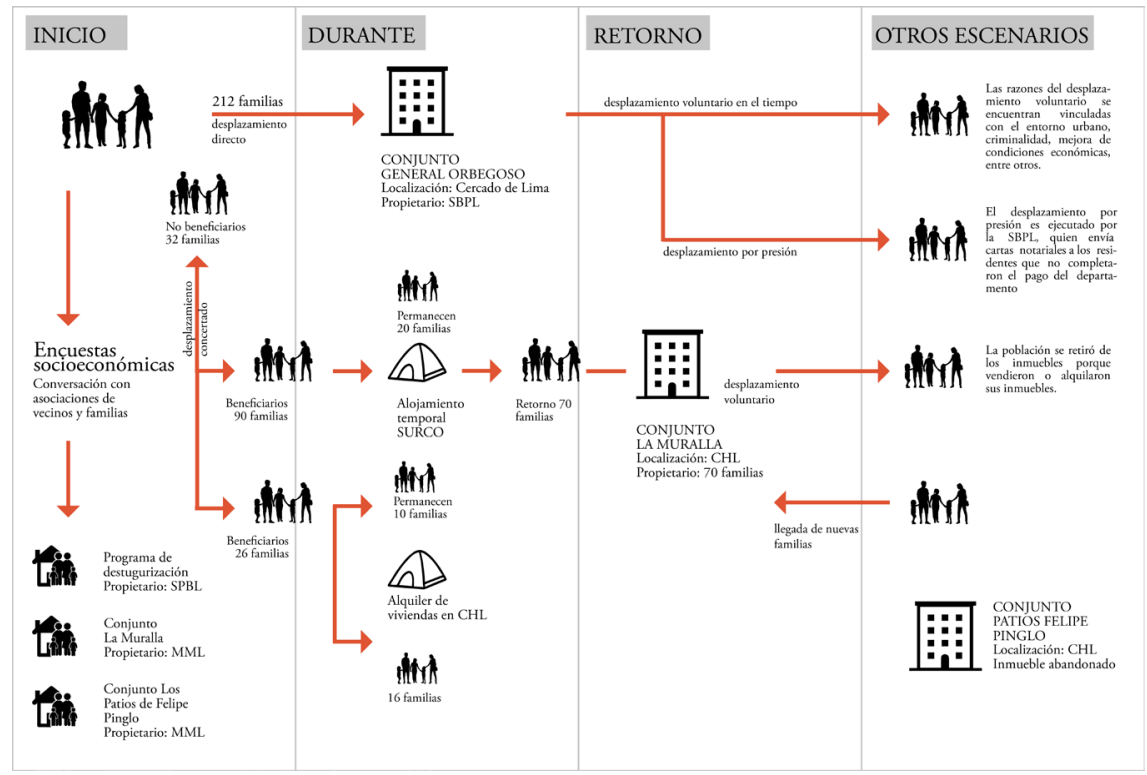

FUENTE: ELABORACIÓN PROPIA

El proceso de desplazamiento ha generado escenarios inesperados. Para el primer caso, además del proceso de desplazamiento, Barrios Altos (BA) - Conjunto Orbegoso, ocurrieron otros procesos de desplazamiento interno, BA - BA. De acuerdo con las entrevistas y conversaciones que se tuvo con vecinos de los inmuebles, se puede verificar que las personas que vivían en Barrios Altos, o familiares, 
pasaron a ocupar los inmuebles abandonados (diez de los inmuebles intervenidos). La vía para saber de los inmuebles abandonados fue la comunicación informal (por parentesco o amistad), hecho que refuerza lo estudiado por Chirinos (1996): los residentes del CHL prefieren quedarse en el lugar, antes que trasladarse a la periferia. En el segundo caso, nuevas familias se encuentran ocupando los departamentos, ya sea por alquiler, traspaso o venta. En el tercer caso, el conjunto habitacional se encuentra bloqueado financieramente por parte del Ministerio de Economía y Finanzas (MEF), debido a incongruencias en su proceso.

\section{Perceptibilidad}

La perceptibilidad, según González et al. (2015), permite identificar y explicitar la mayor o menor visibilidad de los procesos, destacando su grado de obligatoriedad. En los tres casos hubo intensa difusión mediática; sin embargo, los relatos son discordantes, debido a que los programas son mostrados como grandes logros, mientras que la población involucrada no coincide con tal percepción.

En el primer caso, la difusión del proceso fue mediante el diario Expreso ${ }^{2}$ del día 6 de septiembre de 1996, el cual tuvo como titular: "Trasladan a moradores que viven en tugurios". Según el diario, el proceso fue pacífico y concertado: "Han empacado con gran entusiasmo y orden sus enseres y pertenencias, con el fin de enrumbar antes a su nuevo domicilio”. Pero la entrevistada 1 (com. pers., 12 mayo 2016) da una versión diferente: “(...) entonces se metían a tu casa y decían: ¡ya, ya! vamos a llevarlos a tal sitio, vamos a sacar todas sus cosas. Entonces mi mamá que era anciana, y mi hermana que estaba sola, se quedaron sorprendidas". Del cruce de información, es posible verificar que el gobierno publicitaba al proceso como un logro, mientras que la población desplazada lo percibía como arbitrario y violento. En tanto, los dirigentes vecinales que se quedaron en el CHL mencionan que "ellos tuvieron suerte de ser trasladados a un departamento, pero no supieron aprovechar la oportunidad" (Entrevistado 11, com. pers., 14 diciembre 2016).

En el segundo caso, el conjunto habitacional La Muralla, el proceso de desplazamiento tuvo alta difusión en la prensa: era el primer proyecto para los habitantes del CHL, y fue el primero de la MML con aplicación de la Ley 696. Los medios de comunicación tuvieron encabezados como "Una perla de los proyectos del Estado". Hasta el 2010, los medios transmitieron que el proyecto había mejorado la calidad de vida de los habitantes. Igualmente, la municipalidad describió el proyecto como "no solo es una vivienda, es una nueva forma de vida" (Emilima, 2010, p. 30). Los funcionarios municipales entrevistados coinciden en que el proyecto debe ser replicado, pues logró mejorar las condiciones del lugar. Al preguntar sobre los desplazamientos generados durante el proceso, uno de los entrevistados mencionó que "es normal tener desplazamientos durante los procesos de renovación urbana" (Entrevistado 9, com. pers., 18 diciembre 2016).

Es importante aclarar que durante el periodo de gobierno de Alberto Fujimori los medios de comunicación estaban intervenidos, es decir, diarios como Expreso producían noticias sesgadas en favor del régimen de turno. 
La participación de las familias en el conjunto La Muralla sería una de las primeras experiencias innovadoras en la construcción de viviendas en el CHL, aunque, de acuerdo con la información entregada por las entrevistadas, no todas las familias participaron del proceso. La percepción de los beneficiarios revela sus aspectos positivos: "Llegar a las viviendas fue emocionante (...) me siento feliz, es bastante cómodo (...) la comodidad que tengo es que todo está a la mano” (Entrevistada 4, com. pers., 16 diciembre 2016).

Por otro lado, se buscó en los periódicos, El Peruano y El Comercio, alguna nota que mencionara las denuncias de desalojo y la situación de las familias que permanecían en los alojamientos temporales. Al respecto, se encontró la siguiente nota de prensa:

Pese a las condiciones de peligro y miseria en las que viven y a las advertencias silenciosas de sus techos, hay un grupo de vecinos que se resiste a dejar el lugar y rechaza el programa de recuperación que iniciará probablemente la Municipalidad de Lima (...). Según Patricia Santander, el primer bando no quiere ser reubicado porque algunos de sus miembros ya cuentan con otras propiedades. (El Comercio, 5 septiembre 2004)

De los hallazgos y las entrevistas es posible verificar que la exclusión de algunas familias se basa en los siguientes argumentos: contaban con propiedades, se encontraban endeudadas o no habían habitado durante mucho tiempo los inmuebles.

El grupo que no logró retornar al conjunto La Muralla y permanece en los alojamientos manifiesta que los funcionarios les dijeron: "Si usted quiere vivir en su casa, tiene que pagar completo" (Entrevistada 3, com. pers., 18 diciembre 2016). Asimismo, este grupo menciona que "nos prometieron realojarnos (...) no hubo compromiso (...) hay momentos que nos desesperamos (...) nada, hasta ahora. No nos hacen caso" (Entrevistada 5, com. pers., 11 diciembre 2016). El segundo grupo lleva viviendo más de catorce ańos en los alojamientos.

Ante la inoperancia de la MML y EMILIMA, las nueve familias se organizaron para difundir su caso en un programa matutino de televisión, en el que denunciaron: "Nosotros no estamos conformes aquí, porque estamos viviendo mal. Los departamentos ya están acabados, nosotros queremos que se respete como fue la primera etapa: techo propio S/5.000 la cuota inicial” (Panorama Televisión, 2016).

Para el tercer caso, el proyecto tuvo alta difusión en los medios de prensa. EMILIMA creó un canal en YouTube para difundir los avances de las obras, en el cual se menciona: "Construir espacios que mejoren la calidad de vida, posibilitando la revitalización socioeconómica de las familias de extrema pobreza y transformando sus condiciones habitacionales, mediante un verdadero proceso de renovación urbana” (EMILIMA, 2014b).

La percepción de las personas que se encuentran en los alojamientos temporales es de incertidumbre y desesperanza. Al respecto, un exresidente de Cañete 100 mencionó, en una entrevista, que "lo único que pido ahora es que nos digan la verdad, pues estamos cansados de rogar (...). Hemos sido estafados” (Peláez, 2016). Lamentablemente, hasta la actualidad, la MML no se ha pronunciado. Según las entrevistadas, la única vez que accedieron a conversar fue cuando las familias fueron entrevistadas por medios de comunicación. 


\section{Reflexiones finales}

La vivienda tugurizada en los centros históricos del Perú es un problema irresuelto. Los factores que lo explican son diversos; entre ellos, principalmente, las políticas urbanas neoliberales y la concepción de la vivienda como producto inmobiliario en la Constitución Política de 1993. En este sentido, las políticas urbanas y sus estrategias de ejecución, como la renovación urbana, a lo largo de los años han demostrado que no son las más idóneas para resolver el problema de la vivienda. Así, su concepción en la legislación peruana es imprecisa y la forma de aplicarlas, ineficaz, lo cual es verificable en los diversos programas y proyectos ejecutados, que fueron puntuales; es decir, se desarrollaron por lote y no de manera integral; esto es, por manzanas, áreas de intervención o barrios (Riofrío, 2020). Esta producción del espacio urbano central ha generado diversos efectos, destacándose los negativos, como los desplazamientos sociales, la especulación inmobiliaria, el tráfico de terrenos, la reconfiguración de la trama tradicional y la pérdida de actividades culturales tradicionales.

La renovación urbana en el chl se ha convertido en un mecanismo dinamizador del desplazamiento, pues está sostenida en normas que promueven la producción del espacio urbano capitalista en áreas centrales, revalorando áreas para la captura de la rentabilidad urbana (Carlos, 2015; Harvey, 2005), así como ha demostrado ser excluyente y exclusivo. Además de los proyectos de renovación, hay normativas municipales que dan carta libre al desplazamiento, como la Ordenanza Municipal $\mathrm{N}^{\circ} 2267$, del 31 de agosto del 2020. Aunado a ello, los desplazamientos se producen con mayor facilidad debido a que las familias no tienen los recursos suficientes para adquirir un inmueble o pagar el inicial: según lo plantea MVCs, muchas de las familias son pobres o vulnerables, y en su mayoría labora en el sector informal como comerciantes ambulantes. Por tanto, no es una sorpresa encontrar que los desplazamientos, ya sean coercitivos o voluntarios, se presenten en diferentes etapas del proceso de renovación urbana, pues las familias no pueden pagar o no pueden sostenerse mientras los trasladan a los alojamientos temporales.

Los proyectos de renovación urbana y destugurización desarrollados en el CHL fueron aplicados, en los tres casos estudiados, en inmuebles habitados, los que luego fueron demolidos para construir un nuevo conjunto en el lugar. A pesar de ello, la prioridad de los gobiernos nacional y local no estuvo en las mejoras de condiciones de las familias, sino en el rédito político que dichas iniciativas traían al gobierno de turno. Esto es verificable al momento de analizar la difusión los proyectos, pues son publicitados como grandes logros y generadores de mejoras de condiciones, mientras las familias tenían que endeudarse para conseguir el techo propio o, en su defecto, retirarse del CHL.

Finalmente, es importante entender que los desplazamientos sociales no solo son el cambio de las personas de un lugar a otro, sino que implican la pérdida de redes de apoyo, un impacto psicológico y emocional. En este proceso, son las mujeres jefas de familia y los adultos mayores los que se revelan como más vulnerables y perjudicados, pues no cumplen con los requerimientos impuestos por el gobierno y el sistema financiero para la adquisición o arriendo de una nueva vivienda $y$, 
además, son los que sufren más al perder sus redes de apoyo. Ello lleva a insistir en que todo programa de renovación urbana debe priorizar al ser humano por sobre el proyecto, generando protocolos de seguridad y planes de contingencia que protejan a la población afectada. En esta materia, es importante resaltar la labor de las juntas vecinales, en su papel de intercomunicadores y soporte de las familias durante el proceso de renovación urbana.

\section{Agradecimientos}

Esta investigación es parcialmente producto de una tesis de maestría del programa PROURb/UfrJ financiada con la beca de CAPES y FAPERJ. Asimismo, contó con recursos de la Beca de Productividad del CNPq Nivel 2 y Beca Científica de la FAPERJ.

\section{Referencias bibliográficas}

Álvarez, A. \& Mendoza, F. (2016). Renovación urbana y Centro Histórico de Lima: Reflexiones sobre aspectos técnicos en la reconfiguración morfológica de las manzanas 6007 y 6016 de Barrios Altos. Devenir, 3(5), 67-89. https://doi.org/10.21754/devenir.v3i5.288

Carman, M. (2015). Tiempos, políticas y retóricas de ciertos desplazamientos humanos en Buenos Aires. Contested Cities, Working Paper Series, WPCC-15005. http:// contested-cities.net/working-papers/2015/tiempos-politicas-y-retoricas-de-ciertosdesplazamientos-humanos-en-buenos-aires

Carlos, A. F. (Org.). (2015). Crise urbana. Editora Contexto.

Castillo, C. (2015). ¿Gentrificación a la limeña en el centro histórico de Lima? ¿Expulsión o inclusión? 1993-2013. En V. Delgadillo, I. Díaz \& L. Salinas (Coords.), Perspectivas de estudio de la gentrificación en México y América Latina (pp. 133-152). Universidad Nacional Autónoma de México (UNAM).

Chirinos, L. (1996). Barrios céntricos de inquilinato en Lima: los barrios de Monserrate, Barrios Altos y Mendoza Merino. En H. Harms, W. Ludeña \& P. Pfeiffer (Eds.), Vivir en el "centro": vivienda e inquilinato en los barrios céntricos de las metrópolis de América Latina (pp. 17-94). Techniche Universitat Hamburg-Harburg.

Contreras, C. \& Cueto, M. (2018). Historia del Perú contemporáneo. Instituto de Estudios Peruanos.

Contreras, E. (2017). Renovación y desplazamientos urbanos. Surbanistas.

Crestani, A. M. Z. (2015). As faces (in)visíveis da regeneração urbana: rua Riachuelo e a produção de um cenário gentrificado. Cadernos Metrópole, 17(33), 179-200. https:// doi.org/10.1590/2236-9996.2015-3308

Dammert Guardia, M. (2018). Precariedad urbana, desalojos y vivienda en el centro histórico de Lima. Revista INVI, 33(94), 51-76. http://revistainvi.uchile.cl/index.php/INVI/ article/view/1349/1400

De Soto, H. (1986). El otro sendero, la revolución informal. Editorial el Barranco.

Decker, E. (2015). Inquilinato en los tugurios de Lima. Revista WASI, 2(3), 36-58. http://www. revistas.uni.edu.pe/index.php/wasi/issue/view/94 
Delgadillo, V. (2015). Desafíos para el estudio de desplazamientos sociales en los procesos de gentrificación. Contested Cities, Working Paper Series, wPCC-15002. http://contestedcities.net/working-papers/2015/desafios-para-el-estudio-de-desplazamientos-socialesen-los-procesos-de-gentrificacion

Diario Expreso (Lima). (1996, septiembre 6). Trasladan a moradores que viven en tugurios, p. 8A. El Comercio (Lima). (2004, septiembre 4). Casonas serán rehabilitadas con fondos del Ministerio de Vivienda, s. p.

Empresa Municipal Inmobiliaria de Lima (emilima). (2010). Memoria municipal 2000-2010. Municipalidad Metropolitana de Lima.

Empresa Municipal Inmobiliaria de Lima (emilima). (2012, marzo 28). Los patios de Felipe Pinglo (archivo video). Youtube. https://www.youtube.com/watch?v=wAnLk8Iciyo

Empresa Municipal Inmobiliaria de Lima (emilima). (2014a). Memoria municipal 2011-2014. Municipalidad Metropolitana de Lima.

Empresa Municipal Inmobiliaria de Lima (emilima). (2014b). Memoria de gestión 2011-2014. Municipalidad Metropolitana de Lima.

González, A., Blanco, J., Bosoer, L. \& Apaolaza, R. (2015). Movilidades, desplazamientos y territorios: algunos aportes para el debate de la gentrificación. Contested Cities, Working Paper Series, wPCC-15004. http://contested-cities.net/working-papers/2015/ movilidadesdesplazamientos-y-territorios-algunos-aportes-para-el-debate-de-lagentrificacion

Harvey, D. (2005). A produção capitalista do espaço (2a ed.). AnnaBlume editora.

Herrera, C. (2005). Espacios públicos, gestión y renovación urbana en Lima, procesos e impactos. Revista Ur[b]es, 2(2), 171-206.

Janoschka, M. (2016). Gentrificación, desplazamiento, desposesión: procesos urbanos clave en América Latina. Revista INVI, 31(88), 27-71. http://revistainvi.uchile.cl/index.php/ INVI/article/view/1087

Janoschka, M. \& Sequera, J. (2014). Procesos de gentrificación y desplazamiento en América Latina, una perspectiva comparativista. En J. Michelini (Ed.), Desafios metropolitanos, un diálogo entre España y América Latina (pp. 82-104). Catara.

Lagos, W. (2012). Estrategias para la participación social y productiva en renovación urbana de centros históricos. Tesis de maestría, Universidad Nacional de Ingeniería, Lima, Perú. https://1ibrary.co/document/y6jd4ooq-estrategias-participacion-social-productivarenovacion-urbana-centros-historicos.html

Lefebvre, H. (1968). El derecho a la ciudad. Península.

Lozada, L. (2014). Vivienda de interés social en el centro histórico de Lima: Análisis económico de un caso de estudio. Revista WASI, 1(1), 71-90.

Lopez, S. (2017). Los efectos de volver al centro: desplazamientos sociales en el Centro Histórico de Lima (1990-2016). Tesis de maestría, Universidade Federal de Rio de Janeiro, Rio de Janeiro, Brasil.

Ludeña, W. H. (2002). Lima: poder, centro y centralidad. Del centro nativo al centro al centro neoliberal. Revista EURE - Revista de Estudios Urbano Regionales, 28(83), 45-65. http:// dx.doi.org/10.4067/S0250-71612002008300004 
Marcuse, P. (1985). Gentrification, abandonment, and displacement: connections, causes, and policy responses in New York City. Urban Law Annual, Journal of Urban and Contemporary Law, 28(4), 195-240. https://openscholarship.wustl.edu/law_urbanlaw/ vol $28 /$ iss $1 / 4$

Marcuse. P. (1986). Abandonment, gentrification, and displacement: the linkages in New York City. En N. Smith \& P. Williams (Eds.), Gentrification of the city (pp. 110-130). Routledge.

Martí-Costa, M., Duran, G. \& Marulanda, A. (2016). Entre la movilidad social y el desplazamiento. Una aproximación cuantitativa a la gentrificación en Quito. Revista INVI, 31(88), 131160. http://revistainvi.uchile.cl/index.php/INVI/article/view/1092

Mollo, M. (2018). Actores y redes en la gobernanza del centro histórico: el caso de la gestión de recuperación del Rímac. Tesis de maestría, Pontificia Universidad Católica del Perú, Lima, Perú.

Municipalidad Metropolitana de Lima (MML). (1998, enero 21). Ordenanza $N^{\circ}$ 201-Plan Maestro de Lima.

Municipalidad Metropolitana de Lima (MML). (2014). Informe: inmuebles inhabitables, tugurizados, ruinosos total del centro Lima.

Ministerio de Transporte y Comunicaciones (мTC). (1991, julio). Ley N ${ }^{\circ} 696-$ Ley de Promoción a la Inversión Privada en Acciones de Renovación Urbana. Diario El Peruano. https:// leyes.congreso.gob.pe/Documentos/DecretosLegislativos/00696.pdf

Ministerio de Transporte y Comunicaciones (мтс). (1996, agosto 20). Decreto Supremo No 038 de 96. Programa de destugurización de inmuebles en estado ruinoso en el Cercado de Lima y zonas aledañas. Diario El Peruano.

Ministerio de Vivienda, Construcción y Saneamiento (MVCS). (2009, octubre 2). Ley N 29415 de 2009. Ley de Saneamiento Físico Legal de Predios Tugurizados con Fines de Renovación Urbana. Diario El Peruano.

Ministerio de Vivienda, Construcción y Saneamiento (Mvcs). (2010, octubre 30). Decreto Supremo $N^{\circ} 011$ - Reglamento de la Ley de Saneamiento Físico Legal de Predios Tugurizados con Fines de Renovación Urbana. Diario El Peruano.

Olivera, D. (2014). Espacios públicos y renovación urbana del área central de Lima. EST, 1(2), 37-69.

Panorama Televisión. (2016, septiembre 12). Entrevista a Rosario Esquivel. "Familias serán trasladadas por obras de Vía Expresa”. https://www.youtube.com/watch?time_ continue $=1598 \mathrm{v}=\mathrm{vH}$ Hof $960 \mathrm{wiQ}$

Peláez, M. (2016, septiembre 5). Familias exigen a Municipalidad de Lima el hogar prometido. Exitosa. http://exitosanoticias.pe/familias-exigen-a-municipalidad-de-lima-el-hogarprometido/108

Programa Municipal para la Recuperación del Centro Histórico de Lima (prolima). (2019). Plan Maestro del Centro Histórico de Lima al 2029 con visión al 2035. https://aplicativos. munlima.gob.pe/uploads/PlanMaestro/plan_maestro_resumen_ejecutivo.pdf

Riofrío, G. (2020, enero). Vivienda social en el Centro histórico de Lima. Ponencia en Seminario Vivienda social en los centros históricos, Colegio de Arquitectos del Perú, Lima.

Rodríguez, M. (2014). Impacto de las intervenciones en el centro histórico de Lima a partir de la declaración de Patrimonio Cultural de la Humanidad. Devenir, 1(1), 145-168. https://doi.org/10.21754/devenir.v1i1.242 
Rodríguez, I. (2018). Impactos de los catalizadores urbanos en la transformación de los Barrios Altos entre el período de 1535-2015. Devenir, 5(9), 69-82. https://doi.org/10.21754/ devenir.v5i9.200

Rolnik, R. (2015). Guerra dos lugares: A colonizaçâo da terra e da moradia na era das finanças. Boitempo.

Roldán, O. (2017). Gentrificación en centros históricos: una discusión conceptual. Devenir, 4(7), 69-82. https://doi.org/10.21754/devenir.v4i7.136

Uceda, C. (2014). Programa de renovación urbana de El Porvenir 1972-1975. Revista WASI, 1(2), 22-38. 